\title{
Digitized Astronomical Photographic Archives as Large Area Sky Survey of Large Amount of Astronomical Data
}

\author{
René Hudec ${ }^{1,2 * \dagger}$ \\ ${ }^{1}$ Czech Technical University in Prague, Faculty of Electrical Engineering, \\ Technicka 2, CZ 16000 Prague, Czech Republic \\ ${ }^{2}$ Astronomical Institute of the Academy of Sciences of the Czech Republic \\ Fricova 298 - CZ 25165 Ondrejov, Czech Republic \\ E-mail: rene.hudec@gmail.com
}

\begin{abstract}
We report here on an investigation of astronomical plate archives and tests of the scanning devices for digitization of archival astronomical plates. These archives represent huge database of historical astronomical data, astrometric, photometric, and spectroscopic, with many applications in modern astrophysics.
\end{abstract}

Frontier Research in Astrophysics,

26-31 May 2014

Mondello (Palermo), Italy

* Speaker. 


\section{Introduction}

The photography was widely used to record images and spectra in astronomy for more than 100 years. As a result, large collections of astronomical photographic records exist at many worlds observatories and astronomical institutes (Hudec 1999 and 2005). My estimate based on personal visits and works with many different plate collections (more than 50) is that there are more than 7 million astronomical photographic negatives worldwide (Hudec, 1999, Hudec 2005, Hudec and Hudec, 2013).

The basic photographic substrates common in astronomical photographic archives are as follows. (i) Photographic glass negatives and (ii) Photographic negatives on plastic substrates.

There are numerous important astronomical plate archives in many countries, including plate collections that are little known to the community and that have been little investigated in the past. Within the framework of a Czech-US collaborative project (MSMT KONTAKT AMVIS ME09027), we have analyzed some of them, obtaining test scans with the use of a portable digitizing device. Digitization is a necessary step for an extended evaluation of the plate data using dedicated programs and powerful computers. Several recently found large US negative archives are expected to play especially important role in high-energy astronomy. More recently, we investigated European and Chinese astronomical plate archives.

The astronomical plate archives represent the only method how to study the behavior of celestial objects over very long (100 years or even more) time intervals (Hudec, 1999 and 2012). In addition, huge monitoring times allows to detect and to study rare events such as flares. The databases allow to study prominent spectra and/or spectral changes as well (Hudec et al., 2012).

The other division is according to the image/target type recorded on the negative. The basic separation is between direct images and spectral images, where the spectral images can be either single or multiple spectra, or wide field low dispersive spectra with objective prism. The direct images may be single (each star one image) or multiple (each star is recorded several times on the same plate). The negatives were in the past used with large number and rich variety of astronomical telescopes and cameras, and correspondingly, the type variety of archived negatives is also very rich. Many collections represent wide field sky images, but there are also large collections of small negatives, both glass and plastics, taken in the focal plates of medium and large telescopes (Fig. 1,2). While the majority of astronomical negatives worldwide covers sky regions hence with star images, there are also many extended collections with Sun, Moon, and planetary images. In addition to that, on many observatories there are also available additional negatives with buildings, instruments, faces, etc.

The historical astronomical photographic archives represent important part of historical and cultural heritage and, in many cases, reflects and document important epochs of history of science and technology at relevant Institute or Observatory and/or country. Many collections are related to names famous from the history of astronomy and astrophysics.

\section{The science with photographic plates}

The astronomical plates represent important source of valuable data for many areas of recent astronomy and astrophysics. They include huge amount of information as single photographic plate 
may contains up to 100000 star images. The astronomical photographic archives represent the only method how to investigate behavior of celestial objects back in history, and in addition to that, represent huge monitoring intervals necessary to detect rare events such as flashes and flares. Most of plates and negatives were never before investigated in full detail, as before computer era this was virtually impossible. The recent wide plate scanning together with dedicated software allows perfect and complex data mining in these archives for the first time. There are also numerous nonastronomical photographic collections covering various regions of other science and technology.

The photography served as major recording medium not only in astronomy but also in many other areas of science and technology and in addition to that served as medium to record images in national archives, various museum etc. Before era of Xerox copy machines, the photography was the widely used method to make copies of documents, and these negatives are stored in large museum and cultural collections as well. Many problems related to astronomical plate archives such as suitable storage, digitization, defects, etc., are common for all photographic archives.

\section{The astronomical photographic plate archives}

The astronomical archival plate collections that we visited and investigated during last 5 years include those housed in the following 16 US institutions: Carnegie Observatories Pasadena, CA, Lick Observatory, CA Yerkes Observatory, WI, Mt Palomar Observatory, CA, PARI, Rosman, NC (which has a collection of plates from many observatories), KPNO Tucson, AZ, CFHT Waimea, HI, IfA Manoa, HI, USNO Flagstaff, AZ, USNO Washington, DC, Steward Observatory Tucson, AZ, NMSU, Las Cruces, NM, Rosemary Hill Observatory, University of Florida, Gainesville, FL And Leander McCormick Observatory, University of Virginia, Charlottesville, VA, Smithsonial Archives Washington, DC, and Hazy Space Center, Dulles, VA. Our estimate is that there are more than 2 million astronomical archival plates and/or negatives in these archives. There are however many US plate collections not included in our study (Robbins and Osborn, 2009). We performed a quality check and analyzed these plate archives with emphasis on their scientific, historical and cultural value, which we have found to be enormous.

In addition to that, collaboration with several German Institutes with astronomical plate archives started, with emphasis on digitization and investigation of relevant photographic collections. In the recent project, we have visited and digitized samples of astronomical photographic databases e.g. in Torino, Vatican Castel Gandolfo, Catania, Merate, Asiago, Bologna, Loiano, OHP Haute Provence, Nice/Calern, and more recently in China, Red Purple Observatory in Nanjing, and Shanghai/Sheshan.

\section{Photographic plate scanning methods}

Before digitization era, the access to the data recorded in astronomical photographic plates and negatives was difficult. Recently, there are digitization efforts at several observatories, but the data in vast majority of them are still unavailable. The three basic plate scanning techniques developed and tested over last decade are as follows. 
- Custom made high quality scanner, mostly with granite table. Preference: very high astrometric accuracy 0.1 microns or less, drawbacks: very expensive device, expensive operation, plate scanning cost 100 Euro per plate or more.

- Commercial high quality flatbed scanner. Most used instrumentation in plate scanning. Moderately expensive investment and operation, one plate scan typically of order of 10 Euro. Usually good accuracy in one direction but worse in the direction of CCD line movement where typically waves are detectable.

- High quality digital camera with homogeneous light table and high quality lens. Moderate expensive equipment but operation is extremely fast typically one plate just very few seconds and hence very inexpensive. Typical cost of digitization is just 0.5 Euro or even less. Suitable for medium sized up to small and very small plates where the method provides very good resolution and accuracy.

\section{In situ digitization with digital camera}

Most of the plate archives that we visited have no plate scanners and lack modern instrumentation in general. As our study includes plate digitization, it was necessary to find a solution. Since we were going to travel from Europe to the US by air, the obvious option was a transportable digitization device based on a digital camera with a high-quality lens and a stable tripod. This solution has the following advantages over other techniques: the device is easily transportable, and offers much faster scanning and higher repeatability than commercial flatbed scanners, because there are no moving scanner parts. The equipment that we used was as follows: Camera: 21 MPx Canon EOS 5D Mark II, Lenses: Canon EF 24-70 f/2.8 L USM and Canon 70-200mm F4, a stable tripod, and a Fomei LP-310 professional photographic light table. More recently, a better custom-made light table based on highly homogeneous LED illumination was used, and also a further improved camera (39 Mpx) and lenses. The recorded images are then corrected for lens image distortions and for other effects, in order to store research-grade digital images. The achieved pixel size depends on the size of the plate, and fine pixel sizes of order of 25-30 microns or less can be typically obtained for small and medium sized plates (up to $16 \mathrm{~cm}$ x $16 \mathrm{~cm}$ or $13 \mathrm{~cm} \times 13 \mathrm{~cm}$ ) while for larger plates larger pixel sizes will be obtained (or, alternatively, the large plate may be covered by several shots to achieve smaller pixel sizes).

\section{The inventory of astronomical plate collections}

There is no complete inventory of all astronomical photographic records available. The wide field astronomical plate database WFPDB includes only collections wide field sky images (but there are numerous small narrow-field photographic plates as well taken at focal planes of large telescopes before the CCD era) end even that is incomplete. In the USA, the inventory of north American plates was compiled by Robbins and Osborn (2009), but the list is again far from complete. 


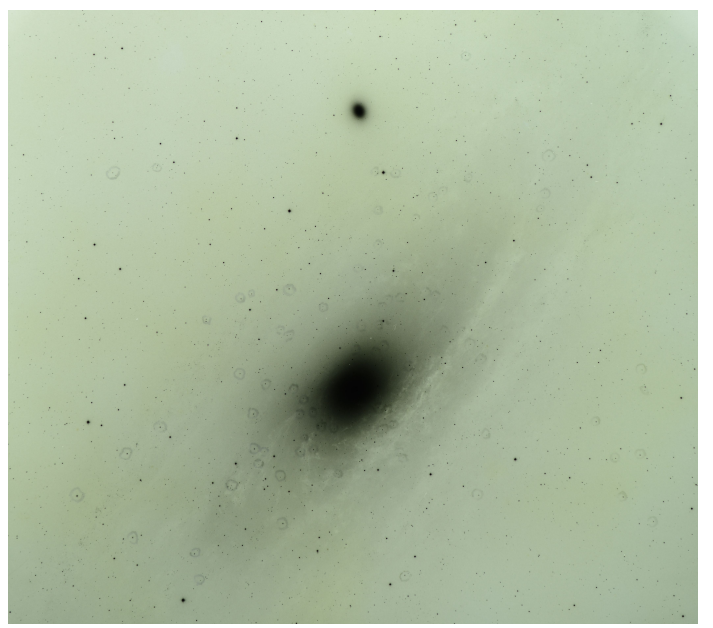

Figure 1: Example of digitized small narrow field direct photographic plate taken in focal plane of large telescope of the Loiano Observatory, image digitized by digital camera, pixel size 5 microns.

Our experience shows that there are numerous astronomical plate collections, some of them large, at places and Institutions unknown to the community before. Some of them were visited by us with test plate scanning using our transportable device.

\section{Conclusions}

The scientific value of historical astronomical data recorded in photographic sky archives is enormous. On the other hand, the digitization efforts are still limited to few places. It is obvious, that, taking the huge number of astronomical archival plates and negatives as well as limited resource into account, we need inexpensive and fast in situ digitization technique.

The alternative plate scanning method based on transportable device was developed and tested. This device was then further improved and upgraded including special software for automated image treatment and lens distorsion correction etc. The astrometric and photometric accuracy of the method was tested and compared, where possible, with other plate scanning methods. The method is fast and inexpensive still yielding scientific grade results.

16 US astronomical plate archives were visited within the AMVIS Czech-US collaborative project. The quality of the plates and their scientific, historical and cultural value were investigated for possible inclusion in the US astronomical plate repository at PARI, NC. Some of these archives (e.g. Baker Super Schmidt, Baker-Nunn, CFHT Waimea, IfA Manoa) were unknown to the astronomical community before our study, and the two first mentioned are expected to play important role in high-energy astronomy because of their very wide-field and very fine time resolution. More recently, additional astronomical plate collections in Europe and China were visited and exploited, with test plate digitization and analysis.

Selected plates were digitized using a transportable scanning device. All the archives that we visited have plates that are scientifically valuable, and in many cases unique. The plates are however often hidden from the astronomical community, and the plates have not yet been catalogued. 
The total number of plates is higher than expected - in many of the locations, the actual number of plates is unknown. As no catalogs exist, the real number of plates is very difficult to estimate, but for sure the places that we visited only in the USA have more than 2 million photographic plates and/or negatives in their collections. We plan to continue these efforts, now with emphasis on German and European astronomical plate collections.

We plan to continue data mining and plate digitization in astronomical photographic plate/negative archives is as follows. (i) Digitize the plate archives using a fast and transportable scanning device, as described above. This scanning method is fast and inexpensive. These are important considerations, as the archives are scattered and there are very large numbers of plates. (ii) Create electronic catalogs. (iii) Include these catalogs into search programs like WFPD, operated by our Bulgarian colleagues (e.g. Tsvetkov et al., 2005, and Tsvetkov, 2009).

\section{Acknowledgements}

We acknowledge GA CR grant 13-39464J. We thanks Dr. Taavi Tuvikene for his help with astrometry accuracy analysis.

\section{References}

[1] Hudec, R. et al., Acta Polytechnica, IBWS2011 Proceedings, 1(52), 2012.

[2] Hudec, R. Astrophysics with Astronomical Plate Archives, in Exploring the Cosmic Frontier: Astrophysical Instruments for the 21st Century. ESO Astrophysics Symposia, European Southern Observatory series. Edited by Andrei P. Lobanov, J. Anton Zensus, Catherine Cesarsky and Phillip J. Diamond. Series editor: Bruno Leibundgut, ESO. ISBN 978-3-540-39755-7. Published by Springer-Verlag, Berlin and Heidelberg, Germany, 2007, p.79

[3] Hudec, R., An introduction to the world's large plate archives, Acta Historica Astronomiae, vol. 6, p. 28-40, 1999.

[4] Robbins, L.; Osborn, W., The Census of Astronomical Photographic Plates in North America, Preserving Astronomy's Photographic Legacy: Current State and the Future of North American Astronomical Plates. ASP Conference Series, Vol. 410. Edited by Wayne Osborn and Lee Robbins. San Francisco: Astronomical Society of the Pacific, 2009., p.81

[5] Hudec, R., World's Astronomical Plate Archives: Past, Present, Future in Virtual Observatory: Plate Content Digitization, Archive Mining and Image Sequence Processing, iAstro workshop, Sofia, Bulgaria, 2005 proceedings, ISBN-10 954-580-190-5, p. 197-208

[6] Hudec R. and Hudec L., Finding Hidden Treasures: Investigations in US Astronomical Plate Archives Acta Polytechnica, Vol. 53, No. 3, p.23, 2013 [4] 